\title{
Is There a Difference in Terms of Perinatal Outcomes Between Fresh and Frozen Embryo Transfers?
}

\author{
Ayse Zehra OZDEMIR', Pervin KARLI²
}

Samsun, Turkey

\section{ABSTRACT}

OBJECTIVE: Nowadays, fresh embryo transfers and frozen embryo transfers are frequently employed in the treatment of in vitro fertilization. This study aims to compare the pregnancy outcomes in patients who underwent fresh embryo transfers and frozen embryo transfers.

STUDY DESIGN: All patients who underwent fresh embryo transfers and frozen embryo transfers at the in vitro fertilization center, Ondokuz Mayis University between 2010 and 2017 were screened retrospectively and the pregnancy results were evaluated at one-year follow-up. The study included a total of 912 transfers, 679 of which were fresh embryo transfers and 233 were fresh embryo transfers, in 756 patients. Comparisons were made in terms of biochemical pregnancy, clinical pregnancy rate, ongoing pregnancy, and live birth rate.

RESULTS: Ectopic pregnancy, biochemical pregnancy, and abortus in fresh embryo transfers were found to be significantly more than that in frozen embryo transfers $(p=0.001)$. However, no statistically significant difference in terms of clinical or ongoing pregnancy rate or live birth rate was observed. Birth weight was significantly lower in fresh embryo transfers than in frozen embryo transfers $(p=0.001, p=$ 0.031). Multiple pregnancies preeclampsia, preterm labor, and placental abruption did not show a statistically significant difference in fresh embryo transfers and frozen embryo transfers. Yet, gestational diabetes was significantly more in frozen embryo transfers $(p=0.011)$.

CONCLUSIONS: Early pregnancy complications in fresh embryo transfers are higher than that in frozen embryo transfers. In terms of neonatal results, higher birth weight and gestational diabetes are more prevalent in frozen embryo transfers. In this study, it has been shown that fresh embryo transfers are more often associated with negative pregnancy outcomes. frozen embryo transfers can be better for pregnancy results

Keywords: Abortus, Fresh embryo transfer, Frozen embryo transfer, Perinatal outcome

Gynecol Obstet Reprod Med 2020;26(2):110-115

\section{Introduction}

The transfer of a frozen embryo by the process of thawing has led to a new era in the history of in vitro fertilization (IVF). At present, embryos can be frozen at all stages right from zygote to blastocyst and can be stored for years (1).

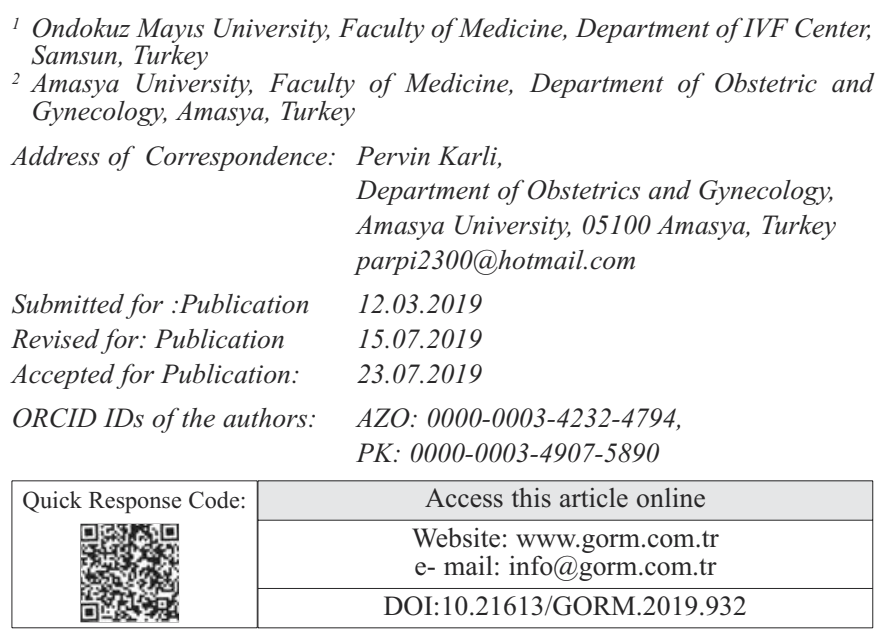

How to cite this article: Ozdemir AZ. and Karli P. Is There a Difference in Terms of Perinatal Outcomes Between Fresh and Frozen Embryo Transfers? Gynecol Obstet Reprod Med 2020;26(2):110-115
The process of Frozen Embryo Transfer (fzET) has enabled the remaining embryos to be stored and then later transferred without the need for new stimulation; thus offering a decrease in the cost of IVF treatment. Additionally, fzET has allowed the postponement of transfers in hyper-responder patients, thereby preventing the occurrence of ovarian hyperstimulation syndrome (OHSS). At the same time, fzET also provides the possibility of a single embryo transfer, thereby reducing the risks associated with multiple pregnancies (2). Besides, it offers a great advantage in cases where the transfer is postponed due to all sorts of medical or social reasons.

Different perinatal outcomes have been observed in fresh embryo transfers (frET) and fzET. Earlier studies show that although fzET increase pregnancy rates, they decrease the low birth weight, small for gestational age (SGA) as well as the preterm risk (3-5). In addition, there are studies showing that it creates macrosomia and increases perinatal mortality (5).

In the fresh cycles, higher progesterone and estrogen levels have a detrimental effect on the endometrium. In fzET, on the other hand, the endometrium is prepared close to natural high estrogen level has been thought to be associated with 
some poor perinatal outcomes (6,7). Supraphysiological steroid levels may be another reason that would explain these perinatal outcomes $(6,7)$.

This study aimed to compare the perinatal outcomes of patients who underwent frET and fzET.

\section{Material and Method}

All patients who underwent frET and fzET in the IVF center of Ondokuz Mayis University between 2010 and 2017 were screened retrospectively and the pregnancy results were evaluated at one-year follow-up. The study was approved by the Ethics Committee, Ondokuz Mayis University. The study was subject to local ethics committee approval (No:11/02/2019-E.3962) and consent for using data. All authors and the study protocol have complied with the World Medical Association Declaration of Helsinki regarding the ethical conduct of research involving human subjects.

A total of 912 transfers, out of which 233 were fzET and 679 were frET, involving 756 patients, were considered. Comparisons were made in terms of biochemical pregnancy, clinical and ongoing pregnancy, and live birth rates. The perinatal outcomes included preterm labor, preeclampsia, placental abruption, and gestational diabetes. Patients with either three or more unsuccessful transfers or those with polycystic ovary syndrome, endometriosis, and known endocrine diseases were excluded from the study.

Babies born under 37 weeks of gestation were considered preterm labor. Pregnancies ending before the 20th gestational week were considered as abortus. Patients with no gestational sac observed although positive beta-human chorionic gonadotropin ( $\beta$-hCG) () were included as biochemical pregnancy. Intrauterine ex patients who did not receive fetal heartbeat after 20 weeks of gestation were taken. The diagnosis of ectopic pregnancy was made by laparoscopy or ultrasonography. Live birth rate was recorded as the birth of a live baby over 20 weeks of gestation. Ongoing pregnancy was considered a pregnancy that continued after the 12 th week. Clinical pregnancy was confirmed by monitoring of fetal heartbeat on the ultrasound.

\section{Statistical analysis}

This study was conducted to determine the effect of fresh or frozen IVF cycles on other variables or parameters. Descriptive statistics for continuous (numerical) variables were expressed as mean and standard deviation, while that for the categorical variables were expressed as number (n) and percentage $(\%)$. In order to determine the sample width (magnitude) of the study, power was taken to be at least 0.80 and Type 1 Error was considered to be 0.05 . Independent t-test was used to compare the mean of continuous variables in the groups. Chi-square test was used to determine the relationship between categorical variables. The statistical significance level (a) was considered to be $5 \%$ in the calculations. The SPSS (IBM SPSS for Windows, Ver. 24) statistical package program was used for carrying out the statistical analysis in the study.

\section{Results}

No statistically significant relationship was found between frET and fzET groups in terms of age $(p>0.05)$ and infertility period $(p>0.05)$. Indications do not show a significant change according to frET and fzET groups $(p>0.05)$. (Table I).

In the groups, 13 patients had ectopic pregnancies in frET, no ectopic pregnancy was detected in the fzET. Ectopic pregnancy was significantly higher in frET group ( $p=0.001)$. Also, abortus and biochemical pregnancy were significantly higher in frET than that in the fzET group $(p=0.001)$. There was no significant difference in terms of intrauterine fetal demise, clinical pregnancy, ongoing pregnancy or live birth rate between the two groups ( $p=1.000, p=0.900, p=0.696, p=0.630$ ) (Table II).

There was no significant difference between preeclampsia, preterm labor and placental abruption between the two groups ( $p=0.440, p=0.706, p=0.865$ ). However, gestational diabetes was significantly more in fzET than in frET babies $(3.9 \%$ vs. $0.7 \%)(p=0.011)$ (Table III).

Table I: Demographic data

\begin{tabular}{|c|c|c|c|c|c|c|}
\hline & & \multicolumn{2}{|c|}{ fzET $(n=233)$} & \multicolumn{2}{|c|}{ frET $(n=679)$} & \multirow{2}{*}{${ }^{*} p$} \\
\hline & & Avg. & SD & Avg. & SD & \\
\hline \multicolumn{2}{|l|}{ Age } & 30.63 & 4.48 & 30.42 & 4.59 & 0.558 \\
\hline \multirow{2}{*}{\multicolumn{2}{|c|}{ Duration of infertility }} & 7.31 & 4.21 & 7.49 & 3.74 & 0.511 \\
\hline & & $\mathbf{n}$ & Column \% & Column \% & $\%$ & ${ }^{* *} p$ \\
\hline \multirow{4}{*}{ Indication } & Unexplained & 128 & 54.9 & 345 & 50.8 & \multirow{4}{*}{0.245} \\
\hline & Low ovarian reserve & 20 & 8.6 & 50 & 7.4 & \\
\hline & Male & 71 & 30.5 & 254 & 37.4 & \\
\hline & Tubal & 14 & 6.0 & 30 & 4.4 & \\
\hline
\end{tabular}

*Independent T-test; ${ }^{* *}$ Chi-square test 
Table II: Pregnancy outcomes in frET and fzET

\begin{tabular}{|c|c|c|c|c|c|}
\hline & \multicolumn{2}{|c|}{ fzET } & \multicolumn{2}{|c|}{ frET } & \multirow{2}{*}{${ }^{*} p$} \\
\hline & $\mathrm{n}$ & $\%$ & $\mathrm{n}$ & $\%$ & \\
\hline Ectopic pregnancy & 0 & 0.0 & 13 & 100.0 & 0.001 \\
\hline Abortus & 40 & 27.8 & 104 & 72.2 & 0.001 \\
\hline Intrautein ex & 2 & 50.0 & 2 & 50.0 & 1.000 \\
\hline Biochemical pregnancy (1) & 43 & 25.1 & 128 & 74.9 & 0.001 \\
\hline Clinical pregnancy & 189 & 81.8 & 533 & 78.7 & 0.900 \\
\hline Ongoing pregnancy & 150 & 64.9 & 430 & 64.0 & 0.696 \\
\hline Live Birth & 147 & 63.6 & 427 & 63.1 & 0.630 \\
\hline Perinatal mortality & 1 & 20.0 & 4 & 80.0 & 0.066 \\
\hline
\end{tabular}

${ }^{*}$ Z-Ratio test result

Table III: Perinatal outcomes in frET and fzET

\begin{tabular}{|c|c|c|c|c|c|}
\hline & \multicolumn{2}{|c|}{ fzET } & \multicolumn{2}{|c|}{ frET } & \multirow{2}{*}{${ }^{*} p$} \\
\hline & $\mathrm{n}$ & $\%$ & $\mathrm{n}$ & $\%$ & \\
\hline Preeclampsia & 6 & 2.6 & 21 & 3.1 & 0.440 \\
\hline Gestational diabetes mellitus & 9 & 3.9 & 5 & 0.7 & 0.011 \\
\hline Placental Abruption & 3 & 1.3 & 6 & 0.9 & 0.706 \\
\hline Preterm Labor & 17 & 11.3 & 58 & 13.3 & 0,245 \\
\hline
\end{tabular}

* Z-Ratio test result

In terms of gestational age, no significant difference was observed between the frET and fzET groups ( $p=0.944$, $p=0.666$ ). Average birth weight was 2838 grams in frET group, while it was 3396 grams in fzET (figure 1).

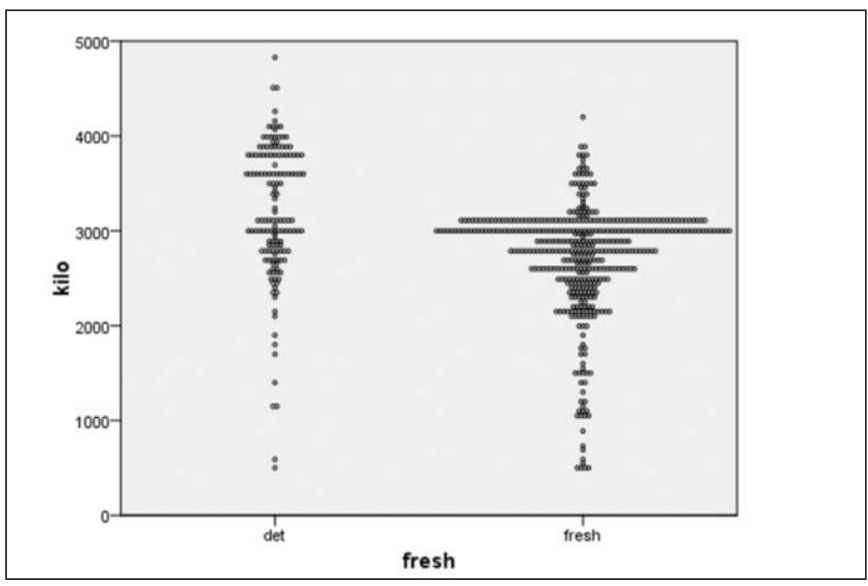

Figure 1: Birth weight in frET and fzET
Birth weight was found to be significantly lower in frET group than that in the fzET group $(p=0.001, p=0.031)$ (Table IV). There was no statistically significant difference in terms of multiple pregnancies between the frET and fzET ( $p=0.389$ ) (Table V). In terms of gender and major congenital anomalies, there was no significant difference between the two groups $(p=0.446)$ (Table VI and VII).

\section{Discussion}

Fresh embryo transfer (frET) and frozen embryo transfer (fzET) are practiced at many centers today. However, differing results related to the neonatal outcomes of these transfers have been obtained from various studies $(2,4,5)$.

It was observed in this study that abortus, biochemical pregnancy, and ectopic pregnancy were significantly higher in frET. However, no significant difference was observed between the two groups when the neonatal outcomes were eval-

Table IV: Comparison of birth weight and gestational age in frET and fzET

\begin{tabular}{|c|c|c|c|c|c|c|}
\hline & & \multicolumn{2}{|c|}{ fzET } & \multicolumn{2}{|c|}{ frET } & \multirow{2}{*}{${ }^{*} p$} \\
\hline & & Avg. & SD & Avg. & SD & \\
\hline Birth Week & singleton & 37.82 & 2.04 & 37.80 & 2.53 & 0.944 \\
\hline \multirow{2}{*}{ (Week Avg.) } & twin & 35.87 & 3.06 & 35.60 & 2.90 & 0.666 \\
\hline & triplet & 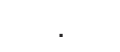 & . & 32.50 & 4.95 & .a \\
\hline Birth & singleton & 3396.1 & 615.4 & 2838.1 & 529.6 & 0.001 \\
\hline \multirow[t]{2}{*}{ Weight (gr) } & twin & 2415.1 & 577.1 & 2164.2 & 516.6 & 0.031 \\
\hline & triplet & . & . & 1600.0 & 777.8 & .a \\
\hline
\end{tabular}


Table V: The relationship between multiple pregnancy rates and types of embryo transfer

\begin{tabular}{|c|c|c|c|c|c|}
\hline & & & fzET & frET & Total \\
\hline \multirow[t]{6}{*}{ Birth Type } & \multirow{2}{*}{ Twin } & $\mathrm{n}$ & 74 & 31 & 105 \\
\hline & & $\%$ & 70.5 & 29.5 & 100.0 \\
\hline & \multirow{2}{*}{ Singleton } & $\mathrm{n}$ & 354 & 114 & 468 \\
\hline & & $\%$ & 775.6 & 24.4 & 100.0 \\
\hline & \multirow{2}{*}{ Triplet } & $\mathrm{n}$ & 2 & 0 & 2 \\
\hline & & $\%$ & 100.0 & 0.0 & 100.0 \\
\hline \multirow[t]{2}{*}{ Total } & & $\mathrm{n}$ & 430 & 145 & 575 \\
\hline & & $\%$ & 74.8 & 25.2 & 100.0 \\
\hline
\end{tabular}

Chi-square $=1.890$

$p=0.389$

Chi-square test

Table VI: Sex rates in frETs and fzETs

\begin{tabular}{|c|c|c|c|c|c|}
\hline & & & fzET & frET & Total \\
\hline \multirow[t]{14}{*}{ Sex } & $\mathrm{m}$ & $\mathrm{n}$ & 154 & 43 & 197 \\
\hline & & $\%$ & $78,2 \%$ & $21,8 \%$ & $100,0 \%$ \\
\hline & $\mathrm{mm}$ & $\mathrm{n}$ & 20 & 4 & 24 \\
\hline & & $\%$ & $83,3 \%$ & $16,7 \%$ & $100,0 \%$ \\
\hline & $\mathrm{mmm}$ & $\mathrm{n}$ & 1 & 0 & 1 \\
\hline & & $\%$ & $100,0 \%$ & $0.01 \%$ & $100,0 \%$ \\
\hline & $f$ & $\mathrm{n}$ & 200 & 71 & 271 \\
\hline & & $\%$ & $73,8 \%$ & $26,2 \%$ & $100,0 \%$ \\
\hline & $\mathrm{fm}$ & $\mathrm{n}$ & 27 & 13 & 40 \\
\hline & & $\%$ & $67,5 \%$ & $32,5 \%$ & $100,0 \%$ \\
\hline & ff & $\mathrm{n}$ & 27 & 14 & 41 \\
\hline & & $\%$ & $65,9 \%$ & $34,1 \%$ & $100,0 \%$ \\
\hline & $\mathrm{ffm}$ & $\mathrm{n}$ & 1 & 0 & 1 \\
\hline & & $\%$ & $100,0 \%$ & $0,0 \%$ & $100,0 \%$ \\
\hline \multirow[t]{2}{*}{ Total } & & $\mathrm{n}$ & 430 & 145 & 575 \\
\hline & & $\%$ & $74,8 \%$ & $25,2 \%$ & $100,0 \%$ \\
\hline
\end{tabular}

Chi-square $=5,802$

$p=, 446$

Chi-square test

uated (except that the birth weight and the frequency of gestational diabetes were more common in the fzET group). The authors believe that these results are either because of asynchronous relation between the endometrium and embryos in frET procedure or the hormonal environment due to Controlled Ovarian Hyperstimulation $(\mathrm{COH})$. Another reason that can explain these results is that the embryos exposed to the freeze-thaw process are stronger. However, according to the results of the present study, application of frET or fzET did not change the perinatal outcomes in the later gestational weeks. Also, the clinical pregnancy rate, live birth rate, and ongoing pregnancy rate were not different.

For better perinatal results, not only a high-quality embryo but also the endometrium needs to be hormonally and biochemically suitable. The studies have shown that high estrogen level caused by $\mathrm{COH}$ may exert its effects on implantation and placenta $(8,9)$. There was no difference found in terms of perinatal outcomes between the frET and fzET patients using donor oocytes. Since similar levels of progesterone and estro- 
gen were found in frET and fzET patients, no endometrial hyperstimulation-induced effects were observed $(10,11)$.

In their study, while evaluating patients using autologous oocytes and donor oocytes, Mar Vidal et al. did not find any difference between the frET and fzET in patients using donor oocytes, yet they found poor perinatal outcomes in patients using autologous oocytes because of $\mathrm{COH}$ administration (12).

The literature does not report any significant difference between frET and fzET in terms of clinical pregnancy and ongoing pregnancy (12). In terms of live birth $(13,14)$, there are studies indicating that live birth rate is higher in fzET, although this result could not be obtained in other studies $(15,16)$. In the present study, there was no difference in terms of clinical pregnancy, ongoing pregnancy, and live birth rate.

Literature also reports different results on abortus. In some studies, abortus has been found to be higher in fzET than that in frET; however; there are also studies stating that there is no difference in terms of abortus between fzET and frET (17-19). Also, the rates of biochemical pregnancy are not different in frET and fzET according to the outcome of some studies (17). Nevertheless, the results of the present study clearly show that abortus and biochemical pregnancy were significantly higher in frET.

Earlier studies on ectopic pregnancy have shown that the frequency of ectopic pregnancy is less in fzET $(20,21)$, which has also been observed in the present study. The presence of high contractility and impaired endometrial receptivity in frET cycles may lead to ectopic pregnancy $(21,22)$.

In terms of neonatal results, 37,703 singleton pregnancies evaluated by Maheshwari et al. in their meta-analysis demonstrated that SGA, low birth weight, preterm labor, perinatal mortality, and postpartum bleeding were less in fzET than that that in frET (4).

In another study, Maheshwari et al. evaluated 112,432 singleton pregnancies and reported that while low and very low birth weight pregnancies were lesser in the fzET group, in terms of preterm rate and anomaly rate, there was no difference between frET and fzET (23). The findings of the present study in terms of neonatal outcomes appear to be consistent with these results. No other neonatal differences were identified other than the birth weight being higher in fzET. Also, gestational diabetes is more frequent in the fzET group; however, the reason is not completely understood. Inadequate growth resulting from incompatibility between the endometrium in the frET cycles and the synchronization with the embryo may be one of the reasons. Furthermore, it is also thought that some changes in the early embryo due to the freeze-thaw process may cause macrosomia in frozen embryos (24).

The authors of the present study did not find any difference between frET and fzET in terms of the gestational week.
However, studies indicating that preterm labor is more common in fzET and that there is no significant difference between a gestational week in frET and fzET have been reported in the literature $(5,25,26)$.

Although the present study is a retrospective one, it is important as it reveals that early pregnancy complications are more frequent in frET.

The most important shortcoming of this study was that the implantation rate was not considered. As the number of embryos transferred in these patients was not reliable in the records of the authors, thus this information was not considered. The present study has shown that the results of early pregnancy are better in fzET than in frET cycles. Deterioration of the endometrial environment with $\mathrm{COH}$ may also have an impact on these results. As for neonatal results, there have been no differences except birth weight and gestational diabetes. Prospective studies are warranted to better understand this issue.

Acknowledgments: Thank you for helping OMU IVF center employees

Authors have declared that they have no conflict of interest We didn't receive funding from any institution.

Author contribution: AZÖ: Constructing an idea or hypothesis for the research, Designing and/or planning methodology, Providing material and enviromental supports, Data collection, Analysis and/or interpretation, Writer, Supervision and critical review. PK: Designing and/or planning methodology, Data collection, Literature review, Writer, Supervision and critical review

\section{References}

1. Sperof L, Fritz MA. Assisted reproductive technologies. In: Mach A Fritz and Leon Speroff, editors. 8th Ed. Clinical gynecologic endocrinology and infertility. Philadelphia: Lippincott Williams \& Wilkins; 2011. p. 1215-1270.

2. Grady R, Alavi N, Vale R, Khandwala M, McDonald SD. Elective single embryo transfer and perinatal outcomes: a systematic review and meta-analysis. Fertil Steril. 2012;97(2):324-31.

3. Pinborg A, Loft A, Aaris Henningsen AK, Rasmussen S, Andersen AN. Infant outcome of 957 singletons born after frozen embryo replacement: the Danish National Cohort Study 1995-2006. Fertil Steril. 2010;94(4):1320-7.

4. Maheshwari A, Pandey S, Shetty A, Hamilton M, Bhattacharya S. Obstetric and perinatal outcomes in singleton pregnancies resulting from the transfer of frozen thawed versus fresh embryos generated through in vitro fertilization treatment: a systematic review and metaanalysis. Fertil Steril. 2012;98(2):368-77.

5. Wennerholm UB, Henningsen AK, Romundstad LB, Bergh C, Pinborg A, Skjaerven Ret al. Perinatal outcomes 
of children born after frozen-thawed embryo transfer: a Nordic cohort study from the CoNARTaS group. Hum Reprod. 2013;28(9):2545-53.

6. Shapiro BS, Daneshmand ST, Garner FC, Aguirre M, Hudson C, Thomas S. Evidence of impaired endometrial receptivity after ovarian stimulation for in vitro fertilization: a prospective randomized trial comparing fresh and frozen-thawed embryo transfer in normal responders. Fertil Steril. 2011;96(2):344-8.

7. Shapiro BS, Daneshmand ST, Garner FC, Aguirre M, Hudson C, Thomas S. Evidence of impaired endometrial receptivity after ovarian stimulation for in vitro fertilization: a prospective randomized trial comparing fresh and frozen-thawed embryo transfers in high responders. Fertil Steril 2011;96(2):516-8.

8. Giorgetti C, Vanden Meerschaut F, De Roo C, Saunier O, Quarello E, Hairion D, et al. Multivariate analysis identifies the estradiol level at ovulation triggering as an independent predictor of the first trimester pregnancy-associated plasma protein-A level in IVF/ICSI pregnancies. Hum Reprod 2013;28(10):2636-42.

9. Pereira N, Reichman DE, Goldschlag DE, Lekovich JP, Rosenwaks Z. Impact of elevated peak serum estradiol levels during controlled ovarian hyperstimulation on the birth weight of term singletons from fresh IVF-ET cycles. J Assist Reprod Genet. 2015;32(4):527-32.

10. Solé M, Santaló J, Boada M, Clua E, Rodríguez I, Martínez F, et al. How does vitrification affect oocyte viability in oocyte donation cycles? A prospective study to compare outcomes achieved with fresh versus vitrified sibling oocytes. Hum Reprod. 2013;28(8):2087-92.

11. Cobo A, Kuwayama M, Pérez S, Ruiz A, Pellicer A, Remohí J. Comparison of concomitant outcome achieved with fresh and cryopreserved donor oocytes vitrified by the Cryotop method. Fertil Steril. 2008;89(6):1657-64.

12. Vidal M, Vellvé K, González-Comadran M, Robles A, Prat M, Torné M, et al. Perinatal outcomes in children born after fresh or frozen embryo transfer: a Catalan cohort study based on 14,262 newborns. Fertil Steril. 2017;107(4):940-7.

13. Chen ZJ, Shi Y, Sun Y, Zhang B, Liang X, Cao Y, et al. Fresh versus frozen embryos for infertility in the polycystic ovary syndrome. N Engl J Med. 2016;375(6):523-33.

14. Coates A, Kung A, Mounts E, Hesla J, Bankowski B, Barbieri E, et al. Optimal euploid embryo transfer strategy, fresh versus frozen, after preimplantation genetic screening with next generation sequencing: a randomized controlled trial. Fertil Steril. 2017;107(3):723-30.

15. Ferraretti AP, Gianaroli L, Magli C, Fortini D, Selman HA, Feliciani E. Elective cryopreservation of all pronucleate embryos in women at risk of ovarian hyperstimulation syndrome: efficiency and safety. Hum Reprod. 1999;14(6):1457-60.
16. Yang S, Pang T, Li R, Yang R, Zhen X, Chen X, et al. The individualized choice of embryo transfer timing for patients with elevated serum progesterone level on the HCG day in IVF/ICSI cycles: a prospective randomized clinical study. Gynecol Endocrinol. 2015;31(5):355-8.

17. Aflatoonian A, Mansoori Moghaddam F, Mashayekhy M, Mohamadian F. Comparison of early pregnancy and neonatal outcomes after frozen and fresh embryo transfer in ART cycles. J Assist Reprod Genet. 2010;27(12):695700 .

18. Aytoz A, Van den Abbeel E, Bonduelle M, Camus M, Joris H, Van Steirteghem A, et al. Obstetric outcome of pregnancies after the transfer of cryopreserved and fresh embryos obtained by conventional in-vitro fertilization and intracytoplasmic sperm injection. Hum Reprod. 1999; 14(10):2619-24.

19. Van Steirteghem AC, Van der Elst J, Van den Abbeel E, Joris H, Camus M, Devroey P. Cryopreservation of supernumerary multicellular human embryos obtained after intracytoplasmic sperm injection. Fertil Steril. 1994;62(4): 775-80.

20. Ishihara O, Kuwahara A, Saitoh H. Frozen-thawed blastocyst transfer reduces ectopic pregnancy risk: an analysis of single embryo transfer cycles in Japan. Fertil Steril. 2011;95(6):1966-9.

21. Shapiro BS, Daneshmand ST, De Leon L, Garner FC, Aguirre M, Hudson C. Frozen-thawed embryo transfer is associated with a significantly reduced incidence of ectopic pregnancy. Fertil Steril. 2012;98(6):1490-4.

22. Jee BC, Suh CS, Kim SH. Ectopic pregnancy rates after frozen versus fresh embryo transfer: a meta-analysis. Gynecol Obstet Invest. 2009;68(1):53-7.

23. Maheshwari A, Raja EA, Bhattacharya S. Obstetric and perinatal outcomes after either fresh or thawed frozen embryo transfer: an analysis of 112,432 singleton pregnancies recorded in the Human Fertilisation and Embryology Authority anonymized dataset. Fertil Steril. 2016;106(7): 1703-8.

24. Pinborg A, Henningsen AA, Loft A, Malchau SS, Forman $\mathrm{J}$, Andersen AN. Large baby syndrome in singletons born after frozen embryo transfer (FET): is it due to maternal factors or the cryotechnique? Hum Reprod. 2014;29(3): 618-27.

25. Ishihara O, Araki R, Kuwahara A, Itakura A, Saito H, Adamson GD. Impact of frozen-thawed single-blastocyst transfer on maternal and neonatal outcome: an analysis of 277,042 single-embryo transfer cycles from 2008 to 2010 in Japan. Fertil Steril. 2014;101(1):128-33.

26. Kato O, Kawasaki N, Bodri D, Kuroda T, Kawachiya S, Kato K, et al. Neonatal outcome and birth defects in 6623 singletons born following minimal ovarian stimulation and vitrified versus fresh single embryo transfer. Eur J Obstet Gynecol Reprod Biol. 2012;161(1):46-50. 